\title{
CCII-based continuous-time filters with reduced gain-bandwidth sensitivity
}

S.-I. Liu

Prof. H.-W. Tsao

J. Wu

\begin{abstract}
Some new MOSFET-C integrators and CMOS current conveyors for realisations of the ladder filters are proposed. These integrators require smaller capacitance than with those currently used. The integrators also provide a better high-frequency performance in terms of gain and phase error responses. A sixth-order bandpass ladder filter, with $460 \mathrm{KHz}$ centre frequency, $0.5 \mathrm{~dB}$ ripple, and ripple bandwidth $120 \mathrm{KHz}$, is designed and simulated. A third-order lowpass filter, with $2 \mathrm{~dB}$ ripple and $50 \mathrm{KHz}$ ripple bandwidth, is experimentally demonstrated. The results reported in the paper will be useful in the realisation of high-frequency integrated continuous-time filters.
\end{abstract}

\section{Introduction}

The continuous-time integrated filter techniques have recently been receiving considerable attention in MOS VLSI technology, because these filters can be stabilised against fabrication tolerances and temperature variations [1]. In certain mixed analogue digital applications, it has been demonstrated that continuous-time filters do offer advantages over other types of integrated filter, such as switched-capacitor filters and digital filters [1]. As in most analogue signal processing, continuous-time MOSFET-C circuits are accomplished through the use of feedback around a high-gain voltage operational amplifier $(\mathrm{OA})$ to achieve a well defined voltage transfer function that is insensitive to the characteristic variations of active devices. Unfortunately, the voltage controlled voltage source OA has several limitations that prevent high-performance operation. One of those limitations is that the product of the bandwidth and the voltage gain is approximately constant [2]. Hence, the operating frequency range of the conventional voltage OA based circuits is inevitably limited. And this also causes deviation from the desirable performance of circuits, leading, in some cases, to the required specifications, not being satisfied.

The advantages of using current amplifier topologies configured to provide closed-loop voltage amplifications are high slew rate and wide bandwidth, which are independent of the closed-loop voltage gain $[3,4]$. In recent years, second-generation current conveyors (CCIIs) have been found useful in many applications. The advantage

Paper 7782G (E10), first received 9th December 1989 and in revised form 28th August 1990

The authors are with the Department of Electrical Engineering, National Taiwan University, Taipei, Taiwan, Republic of China 10764 of the CCIIs in the synthesis of various analogue signal processing circuits has been presented [5]. Although the applications and the methods for realising CCIIs have been presented, however, most of them have been restricted to the bipolar junction transistor technology in the past [6-8].

In this paper, we propose three new MOSFET-C integrators using CMOS CCIIs. Theoretically, these integrators can achieve complete cancellation of MOS nonlinearities of both even and odd modes [9-11] and have a better high-frequency response compared with the OA based integrators [4-6]. Moreover, with the same time constant, two of these integrators will need a smaller capacitance area than conventional ones [9-11].

\section{Circuit description}

Basically, a CCII is a three-port network having the terminal characteristic described by the matrix equation

$$
\left[\begin{array}{c}
i_{y} \\
v_{x} \\
i_{z}
\end{array}\right]=\left[\begin{array}{ccc}
0 & 0 & 0 \\
1 & 0 & 0 \\
0 & \pm k & 0
\end{array}\right]\left[\begin{array}{l}
v_{y} \\
i_{x} \\
v_{z}
\end{array}\right]
$$

where the plus and minus signs of the current transfer ratio $k$ denote positive and negative current conveyors, respectively. The circuit symbol of a CCII is shown in Fig. 1a. Various methods can be used to implement a CMOS CCII. A possible realisation is shown in Figs. $1 b$ and $c$. Let us first consider the CCII + (positive current conveyor), shown in Fig. 1b, which consists of a voltageto-current convertor and a current mirror. Since the input impedance of a MOSFET is high, one can assume the input current $i_{y}$ is zero. Let the currents in the three identical current sources composed of MOSFETs (M7 8), (M13, 15) and (M14, 16), respectively, be $I_{B}$. When there is a small signal current $i_{x}$ flowing out of Port $\mathrm{X}$ the voltage follower will force the currents of $M_{9}$ and $\mathbf{M}_{11}$ to be $I_{B}-i_{x}$ and $v_{x}=v_{y}$. Meanwhile, the current mirror $\mathbf{M}_{10}$ and $\mathbf{M}_{12}$ will copy the same current $I_{B}-i_{x}$. Hence, Port $\mathrm{Z}$ will have a current $i_{z}$ which is equal to $i_{x}$. In general, if the aspect ratios $W / L$ of transistors connected to Port $Z$ (including $\mathbf{M}_{10}, \mathbf{M}_{12}, \mathbf{M}_{14}$ and $\mathbf{M}_{16}$ ) is $k$ times those of transistors connected to Port $\mathrm{X}$, then $i_{2}=k i_{x}$. The operation principle of a CCII-(negative current conveyor) is similar to that of the CCII +, except that another current mirror must be added to invert the polarity of the current $i_{2}$, as shown in Fig. 1c.

For an NMOS transistor biased in the triode region, the channel current is given in terms of several basic MOSFET parameters as [10]

$$
I_{D}=F\left(V_{D}, V_{G}\right)-F\left(V_{S}, V_{G}\right)
$$

IEE PROCEEDINGS-G, Vol. I38, No. 2, APRIL 199 
where $F\left(V_{X}, \quad V_{G}\right)=2 K\left(V_{G}-V_{B}-V_{F B}-\varphi_{B}\right) V_{X}-K\left(V_{X}\right.$ $\left.-V_{B}\right)^{2}-\frac{4}{3} K \gamma\left(V_{X}-V_{B}+\varphi_{B}\right)^{2 / 3}, K=(W / 2 L) \mu C_{o x}$ and $\gamma=\left(1 / C_{o x}\right)\left(2 q N_{A} \varepsilon_{s}\right)^{1 / 2}$.
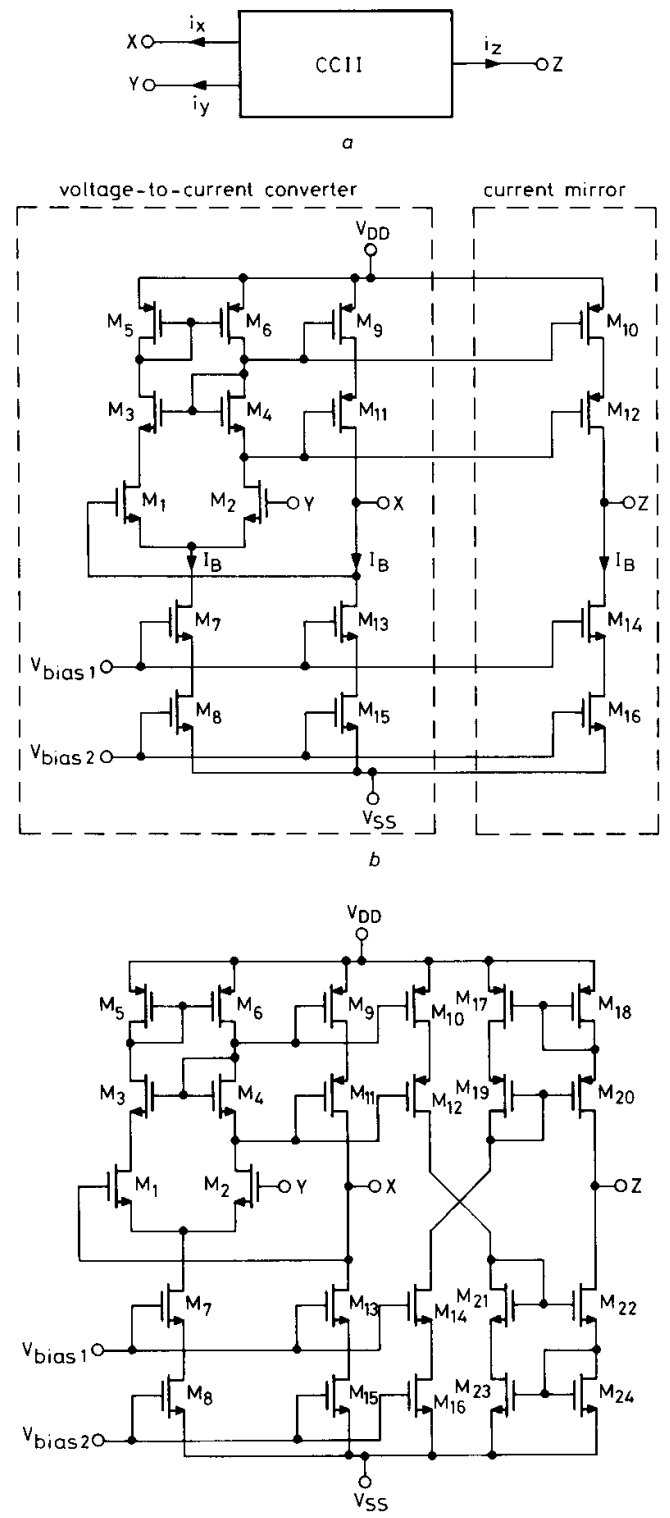

$c$

Fig. 1 Schematic circuit diagrams of the $\mathrm{CCII}+$ and the $\mathrm{CCII}-$ a CMOS CCII symbol

$b$ Simplified CMOS CCII + circuit

c Simplified CMOS CCII - circuil

Czarnul $[10]$ has shown that the MOS resistive circuit (MRC), shown in Fig. $2 a$, can completely cancel the even and odd mode nonlinearities. In the following analysis, it will be assumed that all the transistors in the MRC have the same aspect ratio $W / L$ and operate in the triode region.

Figs. $2 a, b$ and $c$ show the circuit diagrams of the proposed integrators. For the type-A integrator shown in

IEE PROCEEDINGS-G, Vol. 138, No. 2, APRIL 1991
Fig. $2 a$, we can obtain the output current difference of the MRC using eqn. 2 as

$$
\begin{aligned}
I_{1}-I_{2}= & F\left(V_{1}, V_{G A}\right)-F\left(V_{1}, V_{G B}\right) \\
& +F\left(V_{2}, V_{G B}\right)-F\left(V_{2}, V_{G A}\right) \\
= & 2 K\left(V_{G A}-V_{G B}\right)\left(V_{1}-V_{2}\right)
\end{aligned}
$$

Further, the output voltage of the type-A integrator can be expressed as

$$
V_{o u t}=V_{x}+\frac{2}{C} \int i_{z} d \tau
$$

and

$$
V_{y}=\frac{1}{C} \int I_{2} d \tau
$$
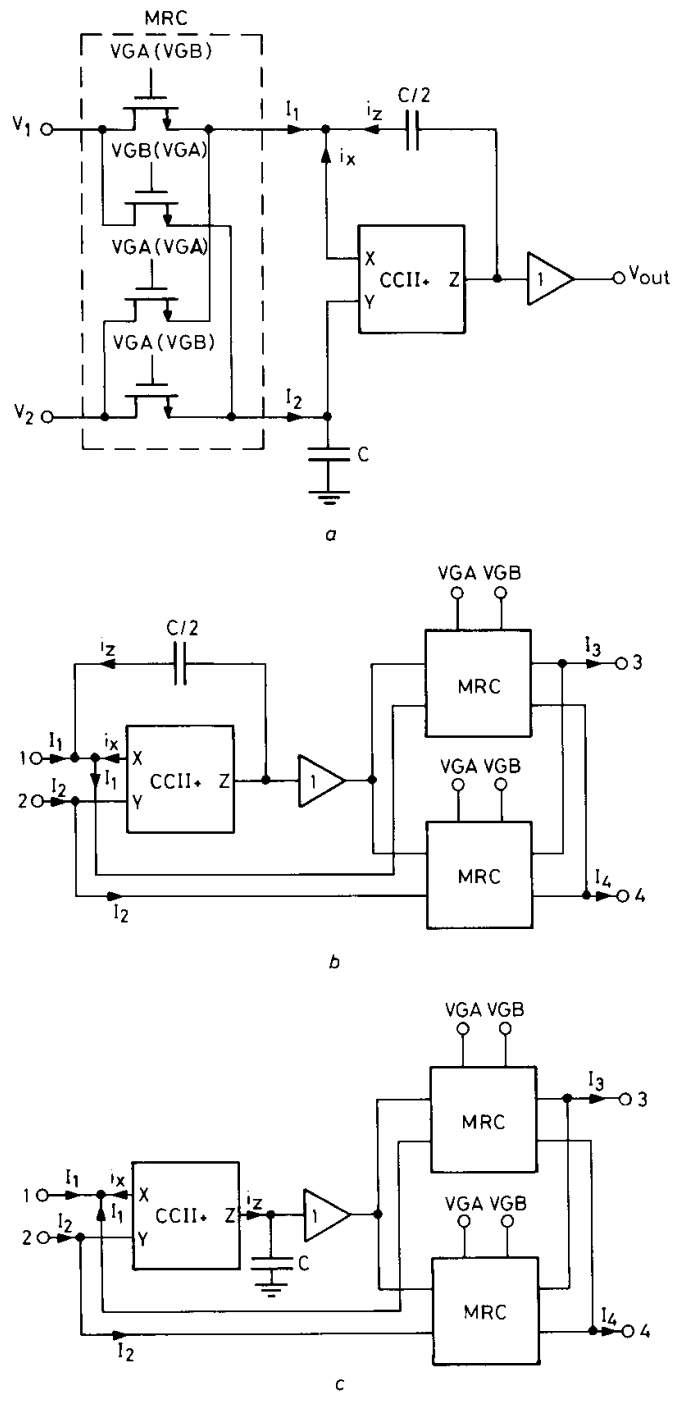

Fig. 2 Integrator circuit diagrams

a Type-A integrator

$c$ Type-C integrator 
Applying KCL at Port X, one can obtain

$$
i_{z}=-\frac{I_{1}}{2}
$$

Therefore, substituting eqns. 3,5 and 6 into eqn. 4 , the output voltage is given by

$$
V_{\text {out }}=-\frac{1}{R C} \int V_{\text {in }} d \tau
$$

where

$$
R= \pm \frac{1}{\mu C_{o x} \frac{W}{L}\left(V_{G A}-V_{G B}\right)}
$$

and $V_{\text {in }}=V_{1}-V_{2}$.

In Fig. $2 a$, the gate voltages outside the parentheses apply to an inverting integrator, whereas those inside apply to a noninverting integrator.

For the type B integrator shown in Fig. $2 b$, we have applied the concept of 'current differential method' proposed in Reference 11. The output current difference $I_{3}-I_{4}$ of the type B integrator can be derived from eqn. 1 as

$$
I_{3}-I_{4}=2 K\left(V_{G A}-V_{G B}\right)\left(V_{z}-V_{x}\right)
$$

The current $i_{z}$ flowing into the capacitor in Fig. $3 b$ is

$$
i_{z}=\left(I_{1}-I_{1}^{\prime}\right) / 2
$$

Because there is no current flowing into Port $Y$, we have

$$
I_{2}=I_{2}^{\prime}
$$

Since the two identical MRCs are under the same bias condition, we also have

$$
I_{1}^{\prime}=I_{2}^{\prime}
$$

Substituting eqns. 10 and 11 into eqn. 9 , one then obtains

$$
i_{z}=\left(I_{1}-I_{2}\right) / 2
$$

Therefore, the output voltage of the type $B$ integrator is

$$
V_{z}=V_{x}-\frac{1}{C} \int\left(I_{1}-I_{2}\right) d \tau
$$

Further, the output current difference $I_{3}-I_{4}$ becomes

$$
I_{3}-I_{4}=-\frac{1}{R C} \int\left(I_{1}-I_{2}\right) d \tau
$$

where $R$ is given in eqn. 8 .

Eqn. 15 indicates that the output current difference $I_{3}-I_{4}$ is proportional to the integration of the input current difference $I_{1}-I_{2}$.

In the same way, one can derive the similar equations for the type $\mathrm{C}$ integrator, shown in Fig. $2 c$, as

$$
\begin{aligned}
& V_{z}=-\frac{1}{C} \int\left(I_{1}-I_{2}\right) d \tau \\
& I_{3}-I_{4}=-\frac{1}{R C} \int\left(I_{1}-I_{2}\right) d \tau
\end{aligned}
$$

These three integrators have different advantages over each other. It is of interest to note that, if the total area of the MRCs of the integrator is fixed, the type B integrator will require half the capacitance $2 C$ that is needed in the original implementation [9-11]. Moreover, if $i_{x}=i_{x} / n$, then the capacitance can be reduced by $n$ times. The same principle can also be applied in the type $A$ and $B$ integrators. The type $\mathbf{C}$ integrator offers a particular advantage because all the capacitors are grounded [12]. Since the type $\mathrm{C}$ integrator does not utilise feedback, it is therefore possible to operate the $\mathrm{CCII}+$ up to its unity-gain frequency.

\section{Performance analysis and comparison}

In the above analysis, we have assumed that the $\mathrm{CCII}+$ and the MOS transistors are ideal. However, several practical considerations may affect the performance of MOSFET-C filters, particularly at higher frequencies where continuous-time techniques may be advantageous. In this Section, we briefly discuss some of the nonideal effects and make comparisons of the frequency responses of the proposed integrators structure, implemented with $\mathrm{CCII}+$, and the conventional ones based on $\mathrm{OA}$ implementation [9-11].

\subsection{Inaccuracy in the $\mathrm{CCll}+$}

The element mismatches, parasitic capacitances and the finite output resistance of the current mirror (i.e. channel length modulation effect) may cause the performance of a practical CCII to deviate from that of an ideal one. First, let us consider the error due to the voltage-to-current convertor. If a resistor $R_{L}$ is connected at Port $X$ of Fig. $1 b$, we can show that the error rate of the transconductance $G_{m}$ of the voltage-to-current convertor can be expressed approximately as

$$
\frac{\delta G_{m}}{G_{m}} \cong \frac{1}{1+R_{L} \frac{g_{m_{9}} g_{m_{1}}}{g_{d s_{6}}}}
$$

where $g_{m_{1}}$ and $g_{m g}$ are the transconductances of the transistors $\mathbf{M}_{1}$ and $\mathbf{M}_{9}$, respectively $g_{d s 6}$ is the drain conductance of the transistor $\mathbf{M}_{6}$. If $R_{L}=10 \mathrm{k} \Omega$ and $I_{B}=86 \mu \mathrm{A}$, then the resulting transconductance error rate is approximately equal to $0.4 \%$. This error rate can be further reduced if a large value of $R_{L}$ is used or the aspect ratios of the transistors $\mathbf{M}_{1}$ and $\mathbf{M}_{9}$ are increased. Secondly, the error rate associated with the current mirror formed by $\mathbf{M}_{9}-\mathrm{M}_{16}$ of the $\mathrm{CCII}+$ can be expressed as

$$
\begin{aligned}
\frac{\delta I}{I}= & \left(\frac{\delta K}{K}-\frac{2 \delta V_{T}}{V_{T}}+\frac{\delta \lambda V_{D S}+\lambda \delta V_{D S}}{1+\lambda V_{D S}}\right)_{M_{15-16}} \\
& \pm\left(\frac{\delta K}{K}-\frac{2 \delta V_{T}}{V_{T}}+\frac{\delta \lambda V_{D S}+\lambda \delta V_{D S}}{1+\lambda V_{D S}}\right)_{M 9-10}
\end{aligned}
$$

where $(\delta K / K),\left(\delta V_{T} / V_{T}\right)$ and $\left(\delta V_{D S} / V_{D S}\right)$ refer to the fractional mismatches in $K$, threshold voltage $V_{T}$, and drainsource voltage $V_{D S}$ of transistors, respectively. $\lambda$ is the channel length modulation coefficient.

\subsection{Effect of finite gain-bandwidth product}

There are two important factors that limit the performance of OA based MOSFET-C integrators: one is the finite gain-bandwidth (GB) product of the OA. The main reason for employing the $\mathrm{OA}$ as the active element is that its virtual ground property enables the cancellation of the nonlinearities of the MOS transistors acting as voltage-controlled resistors. However, the $\mathrm{OA}$ is subject to finite GB effect in the high frequency region. Owing to this reason, the performance of an integrator will deviate from the ideal response in high-frequency operation. In addition, the MOS transistor is not a

IEE PROCEEDINGS-G, Vol. 138, No. 2, APRIL 1991 
lumped resistor as assumed, but rather can be modelled as a uniform RC transmission line in small-signal operation [13]. The distributed capacitance of the MOS transistor can also cause phase error in a similar way to the finite GB of the amplifier. The two-port admittance parameters for a uniformly distributed RC transmission line are given [14] by

$$
Y=\frac{\sqrt{ }(S \tau)}{R_{t} \sinh \sqrt{ }(S \tau)}\left(\begin{array}{cc}
\cosh \sqrt{ }(S \tau) & -1 \\
-1 & \cosh \sqrt{ }(S \tau)
\end{array}\right)
$$

where $\tau$ is the product of the small-signal drain-to-source channel resistance $R_{t}$ and the parallel combination of the channel-to-gate oxide capacitance and the channel-tosubstrate depletion capacitance $C_{t}$. Providing the operating frequency of the circuit is lower than $\omega_{\gamma}=1 / \tau$ of the MOSFET, we can approximate the small-signal transfer characteristics of the type $A$ integrator as

$$
\begin{aligned}
V_{\text {out }}= & \frac{\frac{1}{R_{t_{2}}}-\frac{1}{R_{t_{1}}}}{s C} V_{\text {in }} \\
& +\left(\frac{\frac{S \tau_{1}}{2}+1}{s C R_{t_{1}}\left(\frac{S \tau_{1}}{6}+1\right)}+\frac{\frac{S \tau_{2}}{2}+1}{s C R_{t_{2}}\left(\frac{S \tau_{2}}{6}+1\right)}+1\right) \\
& \times\left(V_{x}-V_{y}\right) \\
\cong & \frac{1}{R_{t_{2}}}-\frac{1}{R_{t_{1}}} V_{i n}
\end{aligned}
$$

where $R_{t_{1}}$ and $C_{t_{1}}$ are the total distributed resistance and capacitance of the MOS transistors connected to $V_{G A}$ in Fig. $2 a$. Similarly, $R_{t 2}$ and $C_{t 2}$ are the total distributed resistance and capacitance of the MOS transistors connected to $V_{G B}$. Eqn. 21 indicates that the output error of this integrator is mainly due to the offset voltage $\left(V_{x}-V_{y}\right)$ of the input stage of the CCII + . As an example, we have compared the gain error and phase error of the integrator in References 9 and 10 and the proposed one in Fig. $2 a$, using SPICE simulation. The simulated unitygain bandwidth of the $\mathrm{CClI}+$, shown in Fig. $1 b$, is about $10 \mathrm{MHz}$. Since it is reasonable to assume the $\mathrm{OA}$ and

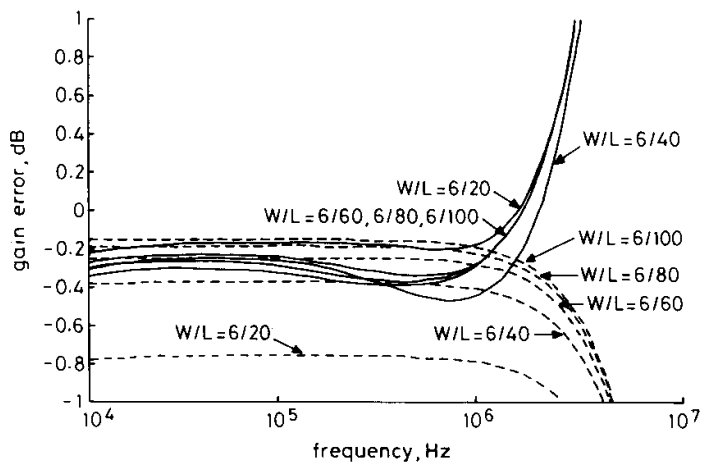

$\mathrm{CCII}+$ have equal GB product in comparing the performance of the integrator, the $\mathrm{OA}$ is assumed with unity-gain frequency $f_{t}=10 \mathrm{MHz}$ and $\mathrm{DC}$ gain $A_{v}=$ 1000. The gain error and phase error of the integrators implemented with $\mathrm{OA}$ and $\mathrm{CCII}+$ are shown Figs. $3 a$ and $b$, with capacitor $\mathrm{C}=10 \mathrm{pF}$ and various aspect ratios $W / L$ of the MRCs. The result shows that the variation range of the gain error is larger for the OA based integrator with the presumed specifications. The integrator using the CMOS CCII has a better phase response in the high-frequency operation. The type $\mathrm{B}$ and $\mathrm{C}$ integrators have also been analysed. The results show that these two integrators have similar characteristics to those of the type-A integrator.

\section{Design examples and optimisation}

A CMOS current conveyor, including junction and layout parasitics, was simulated with $2 \mu \mathrm{m} \mathrm{N} N$-well process parameters. The transistor sizes are listed in Table 1 , and the simulated performances are shown in Table 2. Based on the above building blocks, fully integrated continuous-time MOSFET-capacitor filters using CMOS CCIIs can be constructed. As an example, a

Table 1: Transistor sizes of CMOS current conveyor (Fig 1b)

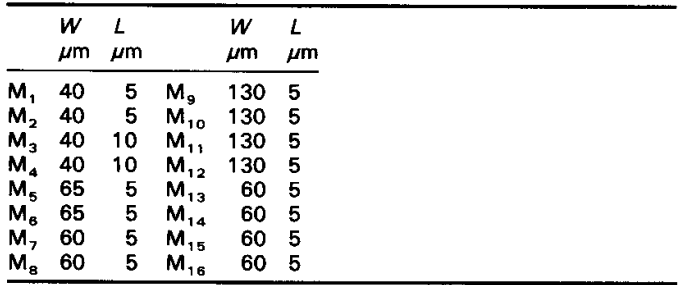

Table 2: Simulated performances of Fig. $1 \mathrm{~b}$ Unity-gain bandwidth $\left(C_{L}=10 \mathrm{pF}\right), \mathrm{MHz} 10$ Slew rate $\left(C_{L}=10 \mathrm{pF}\right)$. $\mathrm{V} / \mu \mathrm{s}$

Output range

Output range, $V$

PSRR $^{+}(0), d B$
PSRR
(0) $(0), d B$

113

THD $\left(@ R_{L} \geqslant 50 \mathrm{~K}, 100 \mathrm{KHz}, \pm 3 \mathrm{~V}\right) \quad 0.014 \%$

(Total harmonic distortion)

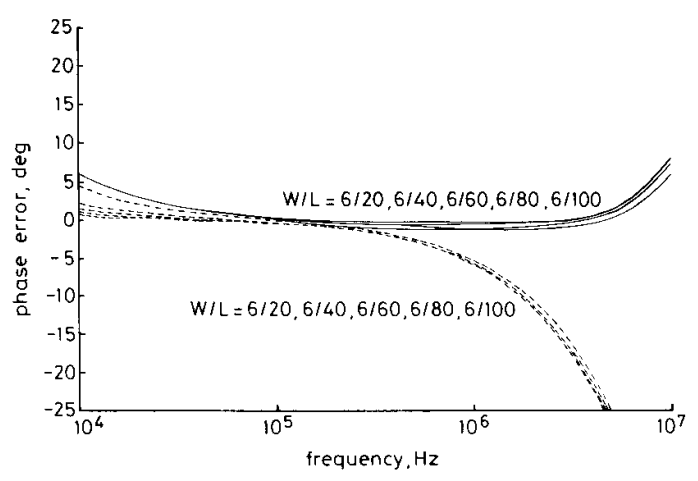

$b$

Fig. 3 Error responses for the $\mathrm{CCII}+-$ based and OA-based integrators [10]

CCII-based

....... OA-based

units: $\mu \mathrm{m}$

a Gain error response

$b$ Phase error responses 
sixth-order doubly terminated ladder filter is designed to have a bandpass response with centre frequency at $460 \mathrm{KHz}, 0.5 \mathrm{~dB}$ ripple in the passband and a bandwidth of $120 \mathrm{KHz}$. In this filter, the type $A$ integrator is used as a basic building block, and its output buffer employs a voltage follower. The circuit is shown in Fig. 4a. According to eqn. 8 , one could use classical results to design the required filter performance to meet the specifications. However, this is based on the assumptions that the effective channel mobility $\mu$ is constant, the parasitic capacitances are negligible, and all the building blocks are ideal. To reduce the deviations caused by nonideal device characteristics, it is desirable to employ iterative numerical optimisation algorithms to redesign the filters [15] The simulated frequency responses of this sixth-order bandpass filter, before and after optimisation, are both shown in Fig. $4 b$. The resulting design parameters are compared, as shown in Table 3.
Table 3: Aspect ratios for MRC in Fig. 4a

\begin{tabular}{lll}
\hline MRC & $\begin{array}{l}\text { Original } \\
\text { design } \\
W / L \\
\mu \mathrm{m}\end{array}$ & $\begin{array}{l}\text { Optimised } \\
\text { design } \\
W / L \\
\mu \mathrm{m}\end{array}$ \\
\hline $\mathrm{MRC}_{1}$ & $6 / 40$ & $6 / 34$ \\
$\mathrm{MRC}_{2,5,6}$ & $6 / 50$ & $6 / 39$ \\
$\mathrm{MRC}_{4,7,8}$ & $6 / 40$ & $6 / 36$ \\
MRC $_{10,12, \mathrm{~L}}$ & $6 / 40$ & $6 / 41$ \\
MRC $_{3}$ & $6 / 40$ & $6 / 36$ \\
MRC $_{9}$ & $6 / 40$ & $6 / 30$ \\
MRC $_{11}$ & $6 / 40$ & $6 / 46$
\end{tabular}

\section{Experimental results}

A third-order lowpass ladder filter has been implemented on a breadboard using the type $\mathrm{C}$ integrators. The schematic diagram of the circuit shown in Fig. $5 a$ is derived

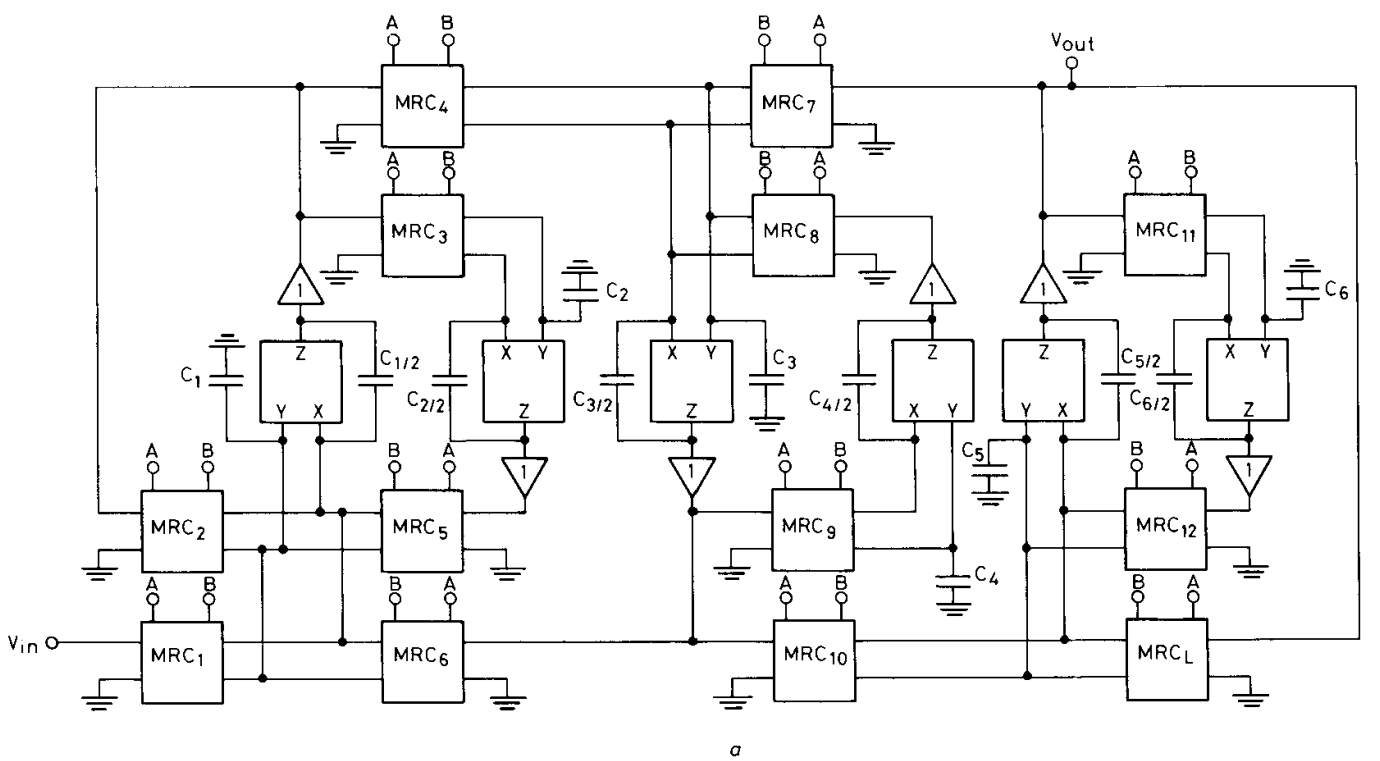

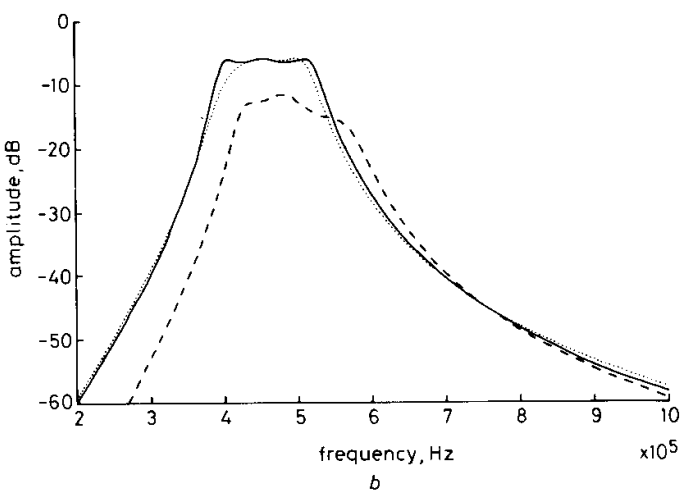

Fig. 4 Sixth-order bandpass ladder filter a Schematic circuit diagram b Simulated frequency response

- ideal response -... before optimisation by the well known signal flow graph technique. This circuit can be further simplified by applying the reduction rules proposed in Reference 11 . The resulting circuit is shown in Fig. $5 b$. This third-order ladder filter just needs 20 MOS transistors as voltage-controlled resistors. The $\mathrm{CCII}+$ circuit was constructed using the bipolar version of CCII $+[4]$ which employed an LF351 OA, together with modified Wilson current mirrors constructed from CA3096AE bipolar junction transistor arrays. The output buffer circuit was realised by a voltage follower with an LF356 OA. The MRC circuits are realised by the PMOS in the CD4007 CMOS transistor arrays. The values of the grounded capacitors in the experiment are $C_{1}=$ $9.7 \mathrm{nF}, C_{2}=4.96 \mathrm{nF}$ and $C_{3}=9.7 \mathrm{nF}$. The gate potentials applied on the gate voltages of the MRCs are $V_{G A}=$ $-5 \mathrm{~V}$ and $V_{G B}=-2.5 \mathrm{~V}$. This filter is realised with $2 \mathrm{~dB}$ ripple and $50 \mathrm{kHz}$ ripple bandwidth. The amplitude and phase responses of this third-order lowpass ladder filter are shown in Fig. 6 . The linearity performance of this filter is illustrated in Fig. 7.

IEE PROCEEDINGS-G, Vol. 138, No. 2, APRIL 1991 

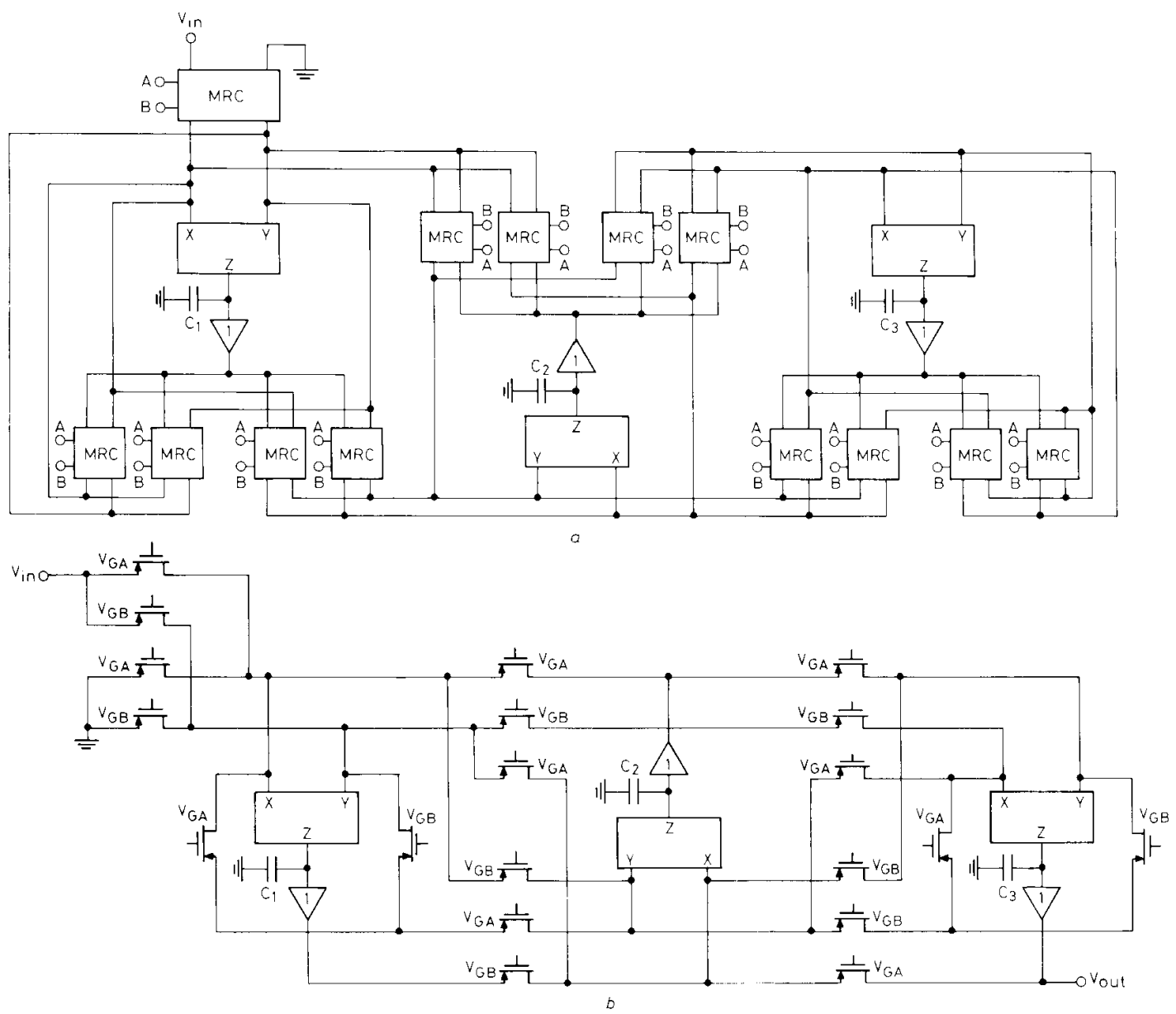

Fig. 5 Third-order lowpass ladder filter

a Circuit diagram

$b$ Simulated frequency response; simplified circuil diagram

$A=V_{\text {GA }}^{\prime}$
$\mathbf{B}=V_{\mathrm{GH}}$
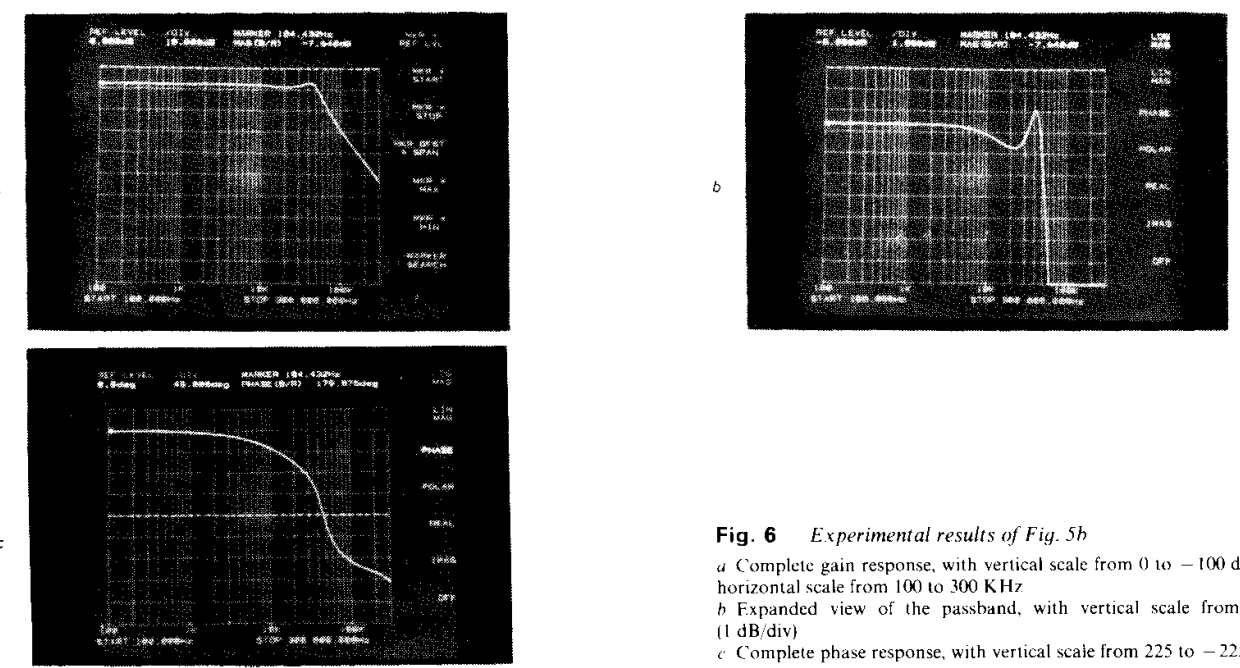

IEE PROCEEDINGS-G, VOL. 138, NO. 2, APRIL 1991

Fig. 6 Experimental results of Fig. $5 \mathrm{~h}$

a Completc gain response, with vertical scale from $010-100 \mathrm{~dB}(10 \mathrm{~dB} / \mathrm{div})$ and horizontal scale from $10010300 \mathrm{KH}$

$b$ Fxpanded view of the passband, with vertical scale from -5 to $-15 \mathrm{~dB}$

(l $\mathrm{dB} / \mathrm{div})$

c Complete phase response, with vertical scale from 225 to -225 (45 div) 


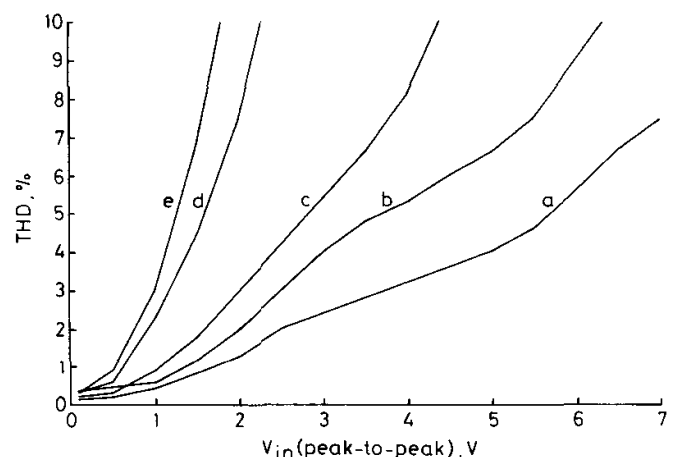

Fig. 7 Total harmonic distortions of Fig. $5 b$ with input signal frequency

$\begin{array}{lll}\text { a } 50 \mathrm{KHz} & \text { c } 25 \mathrm{KHz} & \text { e } 17 \mathrm{KHz} \\ \text { b } 35 \mathrm{KHz} & \text { d } 20 \mathrm{KHz} & \end{array}$

\section{Conclusions}

We have proposed a new CMOS current conveyor and continuous-timeintegrators suitable for high-frequency IC filter applications. The improvement of the frequency response of the integrators using the $\mathrm{CMOS} \mathrm{CCII+} \mathrm{has}$ been presented. A sixth-order ladder bandpass filter is designed using the proposed technique and optimisation method as an example. A third-order lowpass ladder filter is experimentally demonstrated. Both SPICE simulations and experimental results have verified the feasibility of this technique. The proposed method will be useful in the reduction of microprocessor area, and the results also demonstrate an efficient use of the limited frequency response capabilities of active devices to achieve good high-frequency operation.

\section{Acknowledgment}

The authors wish to thank the Electronics Research and Service Organisation (ERSO), of the industrial Tech- nology Research Institute (ITRI) of the Republic of China for supporting this work.

\section{References}

1 BANU, M., and TSIVIDIS, Y.: 'Continuous-time MOSFET-C filters in VLSI', IEEE Trans., 1986, CAS-33, pp. 125-139

2 GRAEME, J.G., TOBEY, G.E., and HUELSMAN, L.P.: 'Operational Amplifiers Design and Applications' (McGraw-Hill, New York, 1971, p. 434

3 ALLEN, P.E., and TERRY, M.B.: 'The use of current amplifiers for high performance voltage applications, IEEE J., 1980, SC-17, pp $155-162$

4 WILSON, B.: 'Constant bandwidth voltage amplification using current conveyor', Int. J. Electronics, 1988, 65, pp. 983-988

5 TOUMAZOU, C., LIDGEY, F.J., and CHEUNG, P.Y.K "Current-mode analogue signal processing circuits-A review of recent developments'. Proc. IEEE Int. Symp. Circuits and Systems, 1989, pp. 1572-1575

6 WILSON, B.: 'High-performance current conveyor implementation', Electron. Lett., 1984, 20, (24)

7 SURAKAMPONTORN, W., and THITIMAJSHIMA, P.: Integrable electronically tunable current conveyors', IEE Proc. G. Electron. Circuits and Syst., 1988, (2)

8 WADSWORTH, D.C.: 'Accurate current conveyor integrated circuit', Electron. Lett., 1989, 25, pp. 1251-1252

9 ISMAIL, M., SMITH, S.V., and BEALE, R.G.: 'A new MOSFET-C universal filter structure for VLSI', IEEE $J$., 1988, SC-23, pp. 183-194

10 CZARNUL, Z.: 'Novel MOS resistive circuit for synthesis of fully integrated continuous-time filters', IEEE Trans., 1986, CAS-33, pp 718-721

11 TAKAGI, S., FUJII, N., and YANAGISAWA, T.: 'A canonical continuous-time MOSEFET-capacitor filter with high linearity'. Proc. IEEE Int. Symp. Circuits Syst., 1988, pp. 2177-2180

12 BHUSAN, M, and NEWCOMB, R. W.: 'Grounding of capacitors in integrated circuits', Electron. Lett., 1967, 3, pp. 148-149

13 KHOURY, J., and TSIVIDIS, Y.: 'Analysis and compensation of KHOURY, J., and TSIVIDIS, Y.: 'Analysis and compensation of
high-frequency effects in integrated MOSFET-C continuous-time high-frequency effects in integrated MOS

14 GHAUSI, M., and KELLY, J.: 'Introduction to DistributedParameter Network, with Applications to integrated circuits' (Holt, Rinehardt and Winston, New York, 1968)

15 LIU, S.I., and SHYU, J.M.: 'Optimization-based design for MOSFET-capacitor filters' 1989 Eectronics Device, Materials, and Circuit Technology Symposium (EDMS) in R.O.C. 